\title{
The Threshold Conditions in Electrical Excitation of the Nerve Fiber. Part II $^{1}$ \\ By \\ ichiji Tasaki \\ Physiological Institute, Keio University, and \\ Tokugawa Biological Institute, Tokyo
}

Received July 21, 1949

In Part I of this article, the process of excitation by brief stimulating currents was discussed. In Part II, we will try in the first place to formulate the threshold conditions for excitation by long, slowly varying current. Then, we will turn to the problem of electrotonus and of repetitive excitation $b_{y}^{r}$ constant current.

\section{Excitation by slowly varying voltages}

1.1 Excitation by linearly rising voltages. It is simple matter to apply, to a nerve fiber, voltages rising linearly at various rates and to record action currents from the site of stimulation. When the voltage increases at a rate smaller than a certain limit which is characteristic of the preparation used, we find that the stimulus fails, as is well known, to excite the fiber. With voltages of steeper gradient, it is observed that an action current is evoked a few milliseconds after the stimulating voltage has reached the level of the rheobase (Fig. 1). ${ }^{2}$

When these linearly increasing voltages are made to change into constant voltages after they have reached certain final levels, it is observed that the threshold final level is perfectly independent of the rate of rise, provided that the rate is greater than the minimal voltage gradient (Fig. 2). Thus, in the diagram showing the relation between the final voltage $v$ and the time of voltage rise $z$, the points representing effective stimuli are limited by the two straight lines I and II in Fig. 2. This point has not been clearly demonstrated in the previous experiments with entire nerve trunks.

Experimental facts presented in this section indicate undoubtedly that, in excitation by long voltage pulses, the rheobasic voltage $\beta$ and the minimal voltage gradient $\mu$, both characterizing the state of the node of

1 This research was supported by a grant from the Ministry of Education.

2 According to Skogland, cited by Granit (1949), the results obtained with ordinary nerve-trunks are considerably different from those stated in this article. The main causes for the difference are considered to be (1) the deformation of the imposed current by the connective tissue envelope and by inactive fibers, (2) the confusion resulting from excitation of different fibers, and (3) the deformation of the effective current due to electrotonic spreading of the current along the fiber which occurs when the stimulating voltage is applied to a region of a fiber including more than one internodal segment (Tasaki, 1939). 
Ranvier under investigation at that moment, play important parts. In the normal motor nerve fiber of the toad, $\beta$ is generally between 20 and 40 $\mathrm{mV}$. The value of $\mu$ varies considerably from preparation to preparation ; it is in most cases between $0.1^{\prime}$ and $1.0 \mathrm{mV} / \mathrm{msec}$.
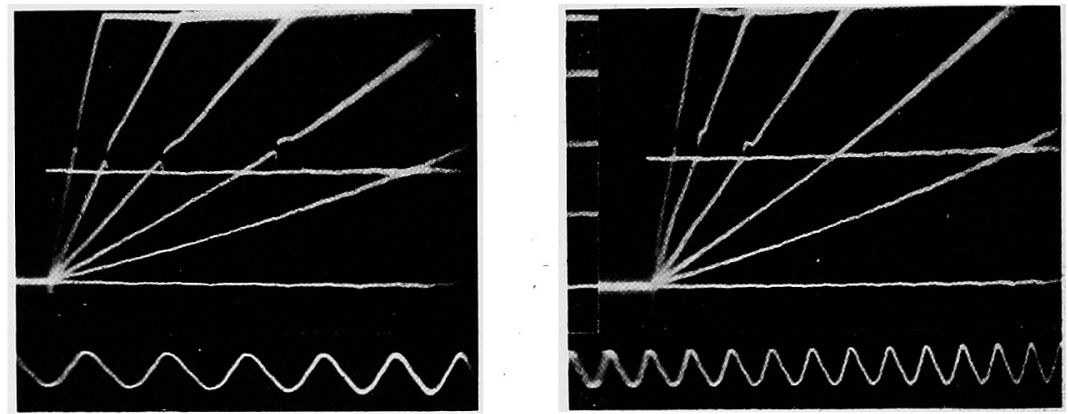

Fig. 1. Superposed oscillograph records showing the relation between the latent period of action current and the rate of voltage increase in excitation of a toad's motor nerve fiber by linearly rising voltages. Right and left photographs were taken from two different preparations. $8^{\circ} \mathrm{C}$. Linearly rising voltages, obtained by the use of a pentode, were applied directly to one of the deflection plates of a cathode ray oscillograph and were tapped to apply to the nerve fiber. The action currents, led from the site of stimulation, were amplified by means of an amplifier coupled with condensers of a small capacity and were led to the other deflection plate. The zero and rheobasic levels of the stimulating voltage are shown by the horizontal lines. The interval between the marks in the middle corresponds to apotential difference of 25 millivolts in the stimulating circuit. The time marker, 50 cycles per second.

1.2 Excitation by exponentially rising voltages. The time course of an exponentially rising voltage can be expressed by

$$
V(\mathrm{t})=v\left(1-e^{-t i r c}\right),
$$

where $v$ represents the final voltage and $r c$ the time constant of voltage rise. If one fixes $r c$ at various values and increases $v$ step by step, one can determine the threshold final voltage $v$ as a function of the time

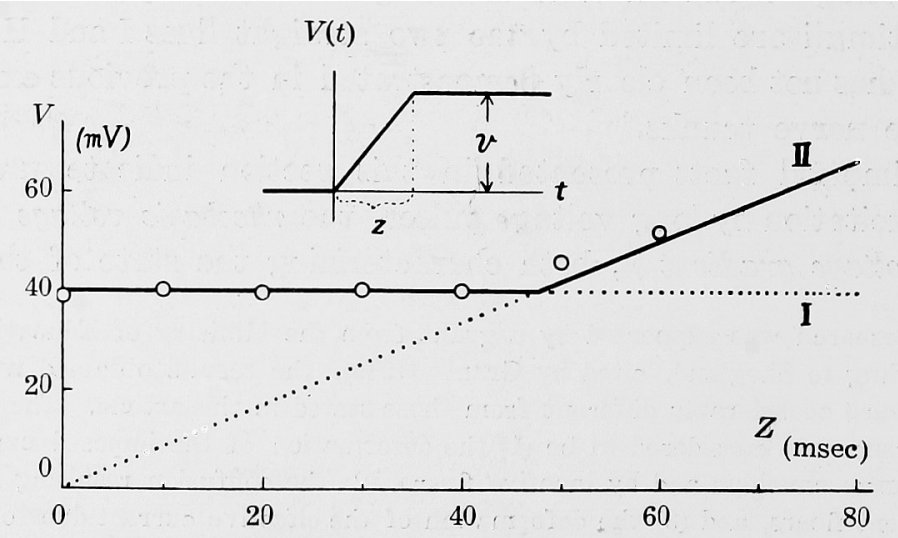

Fig. 2. Relation between ascending time and threshold final level of a linearly rising voltage. A toad's motor nerve fiber at $17^{\circ} \mathrm{C}$. (Tasaki, 1944). 
constant rc. The continuous curves in Fig. 3, A and B, show the time course of such exponentially rising voltages. The discontinuities on the curves indicate the moments at which action currents were observed at the site of stimulation when the fiber was excited by these stimuli.

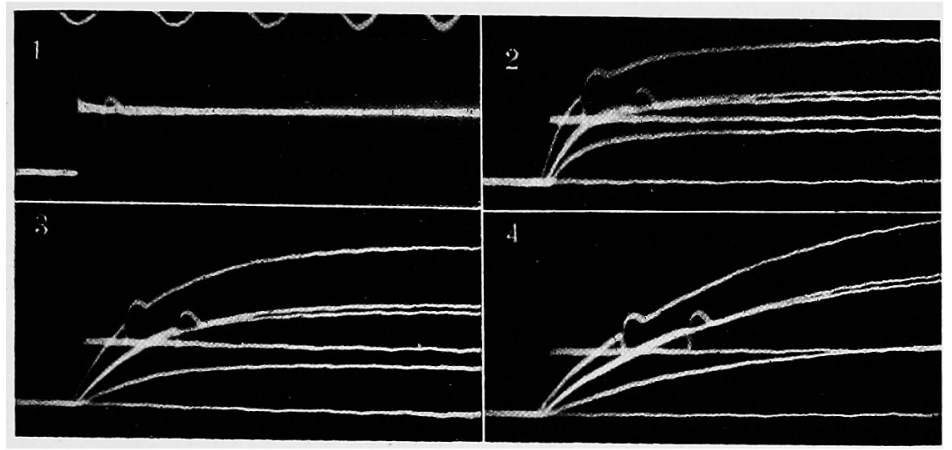

Fig. 3 A. Superposed oscillograph records showing the time courses of exponentially rising stimulating voltages and the action currents led from the site of stimulation. A motor nerve fiber of the toad, $8^{\circ} \mathrm{C}$. (1) Rectangular pulse of $38 \mathrm{mV}$, rheobase. (2) The time constant of voltage increase, $7.7 \mathrm{msec}$; the final voltages, $70,48,45$ and $30 \mathrm{mV}$. (3) $15 \mathrm{msec}$; 80, 56, 53 and $30 \mathrm{mV}$. (4) $30 \mathrm{msec}$; 95, 71, 68 and $34 \mathrm{mV}$. The time marker, 50 cycles per sec.

One finds in these figures immediately that, in threshold excitation, the curves intersect the level of the rheobase $\beta$ at the same gradient and that nerve impulses are released soon after the stimulating voltages have arisen above this level. For very great $r c$ values, exponentially rising voltages can approximately be regarded as linearly rising voltages. It is therefore obvious that, in threshold excitation, the exponentially rising voltages cross the rheobase $\beta$ at a constant gradient $\mu$.
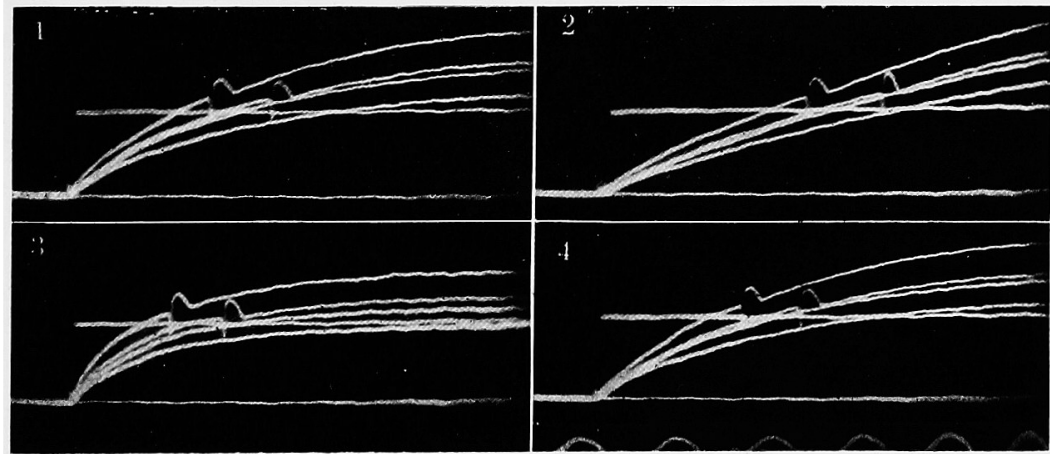

Fig. 3 B. Effect of exponentially rising voltages upon a tonic muscle afferent fiber, $8^{\circ} \mathrm{C}$. (1) 30 msec.; 72, 60, 57 ard $47 \mathrm{mV}$. (2) $65 \mathrm{msec}$; 90, 76, 72 and $61 \mathrm{mV}$. (4) $15 \mathrm{msec}$; $60,50,46$ and $39 \mathrm{mV}$. (4) 30 msec.; 70, 58, 55 and $46 \mathrm{mV}$. The horizontal line represents the level of the rheobase of $41 \mathrm{mV} .50$ cycles per sec.

From eq. (1), it is clear that the time $h$ at which such a stimulating voltage reached the rheobase $\beta$ is given by

$$
\beta=v\left(1-e^{-h / r r}\right) \text {, }
$$

Cytologia 15, 1950 
and the rate of voltage rise at this moment by

$$
\left(\frac{d V(t)}{d t}\right)_{t=h}=\frac{v}{r c} e^{-h / r c}=\mu .
$$

Elliminating $h$ from these two equations, we have

$$
v=\mu r c+\beta,
$$

which the exponentially rising voltage (1) must satisfy if it is to just excite the fiber. In fact, the relation of eq. (4) holds good with amazing accuracy for the data obtained with single fiber preparations and less accurately for the data obtained with ordinary nerve trunk preparations (Cardot and Laugier. 1913; Schriever, 1932 ; Solandt, 1936; Suzuki, 1937).

3.3 Excitation by slowly varying voltages in general. We may generalize the conclusion reached in the preceding two sections as follows: In threshold excitation by any kind of slowly varying voltages, the stimulating voltage surpasses the rheobase $\beta$ at a constant gradient $\mu$. Denoting the slowly varying voltage by $V(t)$, we may express the statement above by the simultaneous equations

$$
V(h)=\beta \quad \text { and } \quad\left(\frac{d V(t)}{d t}\right)_{t=h}=\mu .
$$

Elliminating the parameter $h$, we obtain the equation of threshold excita-
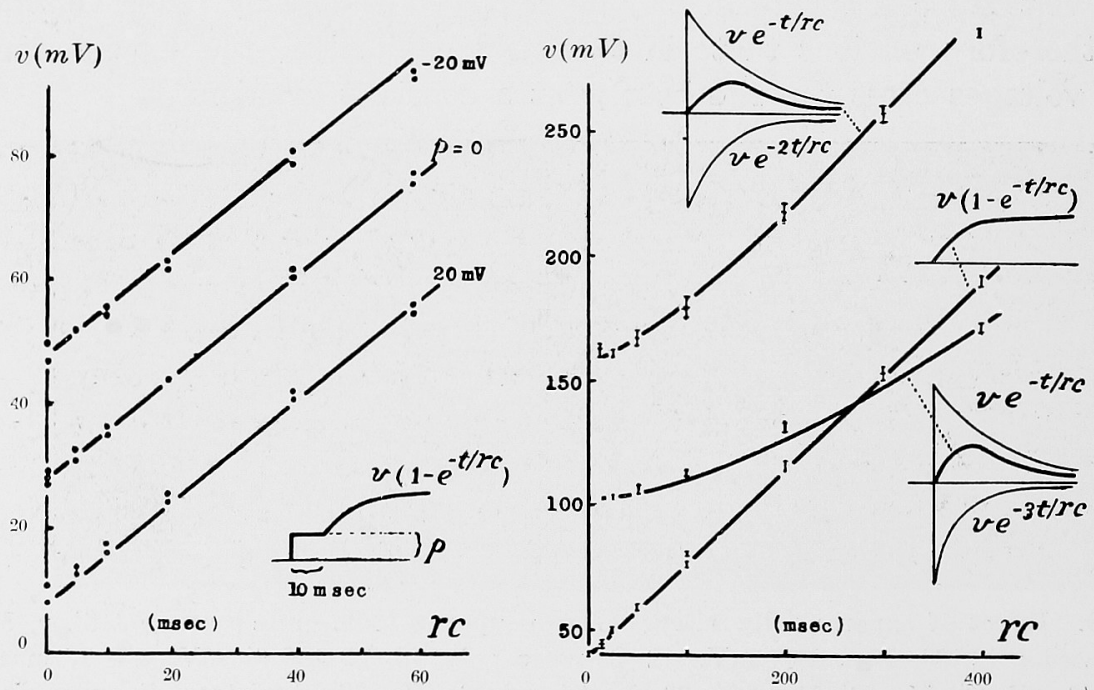

Fig. 4. Left: Relation between threshold strength and rate of rise of an exponentially increasing voltage superimposed to a constant voltage. The exponentially rising voltage was started at $10 \mathrm{msec}$. after the make of the constant voltage. Toad's motor fiber at $60^{\circ} \mathrm{C}$. (Tasaki and Mizutani). Right: Relation between threshold strength and duration of a voltage pulse obtained by double or single condenser charge. Toad's motor nerve fiber at $13^{\circ} \mathrm{C}$. (Tasaki and Bun). 
tion for that particular shape of stimulating voltage.

In case where

$$
V(t)=v\left(1-e^{-t / m}\right)+p,
$$

the equation of threshold excitation becomes

$$
v=\mu r c+\beta+p
$$

which, if $p=0$, reduces to eq. (4) in the preceding section. An example of the experimental results furnished in Fig. 4, left, shows that this equation describes the actual data very well.

When, in the next place, the stimulating voltage is given by

$$
V(t)=v\left(e^{-t / r e}-e^{-n t r c}\right),
$$

where $n$ is a positive integer other than 1 we obtain the equation of threshold excitation

$$
v=\frac{1}{n-1} \sqrt[n-1]{\frac{(n \beta+\mu r c)^{*}}{\beta+\mu r c}},
$$

and that this equation agrees perfectly with the observed data is shown thoroughly in a large number of cases (Tasaki and Bun, see the example of Fig. 4, right).

With a low frequency alternating voltage

$$
V(t)=v \sin 2 \pi f t,
$$

$f$ being the frequency, the equation of threshold excitation becomes

$$
v^{2}=\beta^{2}+\mu^{2} / 4 \pi^{2} f^{2} .
$$

This is practically the same with the equation derived by Hill (1936) and has been shown to agree with actual data (Solandt, 1936).

With isolated single nerve fibers, Bun (unpublished) has carried out very careful experiment with A.C. of below 10 cycles per sec. For comparison of the observed $v-f$ relation with eq. (11), he measured, before and after excitation by A.C., the rheobase and the minimal gradient of the preparation under observation. He saw that the agreement between predicted and observed data was qualitatively satisfactory. We attributed the slight discrepancy to the electrotonic change of $\mu$ during stimulation.

1.4 Effects of various agents upon the minimal gradient and the rheobase. As has been well known, an increase in the Ca-concentration in the surrounding medium of a nerve fiber increases both $\mu$ and $\beta$. A reduction of Ca-ion decreases $\mu$ but affects $\beta$ only slightly. Lowering the temperature decreases $\mu$ but does not change the rheobase of a single nerve fiber appreciably.

It should be born in mind that the change in the minimal gradient $\mu$ by these chemical or physical agents shows a remarkable hysteresis: The effect of these agents remains to persist for some time after the pre- 
paration has been brought back to its original environment (Tasaki, 1949).

Among all the physical agents affecting $\mu$ and $\beta$ of the nerve fiber, the effect of electrotonus seems to us most interesting. Fig. 5 shows the result of an experiment in which trials to determine the thresholds were done about 5 minutes after the start of electrotonus. As has been well

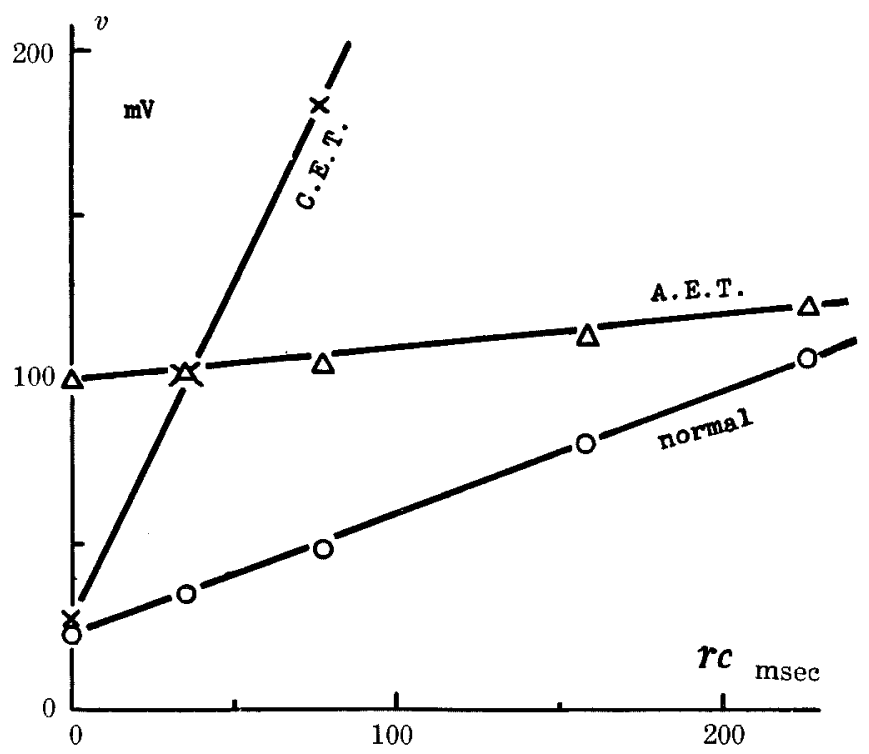

Fig. 5. Relation between threshold strength and rate of rise of an exponentially rising voltage applied to a toad's motor nerve fiber under electrotonus $\left(60 \mathrm{mV}\right.$ ). $5^{\circ} \mathrm{C}$. (Tasaki and Sakaguchi).

known, $\mu$ is markedly increased by catelectrotonus (C.E.T.) and decreased by anelectrotonus (A.E.T.). It has been fully demonstrated that, in excitation of a node under electrotonus, nerve impulsses are released according to the condition stated above, although the values of $\mu$ and $\beta$ are seriously affected by the procedure (Tasaki and Sakaguchi).

The curve $b$ in Fig. 6 shows the threshold strengths for a rectangular voltage pulse superimposed to polarizing voltages of varying strengths. An interpretation of this curve will be presented below.

\section{Excitatory state set up by a slowly varying voltage}

2.1 A triangular test pulse. It has been pointed out already that superimposition of a long triangular voltage pulse of half the threshold strength on a constant voltage of half the rheobase may result in excitation of the nerve fiber (see Part I, Fig. 12, right). This fact indicates, according to our conception of e.s. stated in the previous chapters, that, in threshold excitation by these two kinds of voltage pulses, one and the same "sort" of e.s. plays the role to elicit the impulse. We may denote 
the magnitude of this sort of e.s. by $E_{s}$. We should then be able to measure $E_{s}$ at any moment by means of a triangular test pulse, just as we did in the measurement of $E_{b}$ by means of a brief test shock.

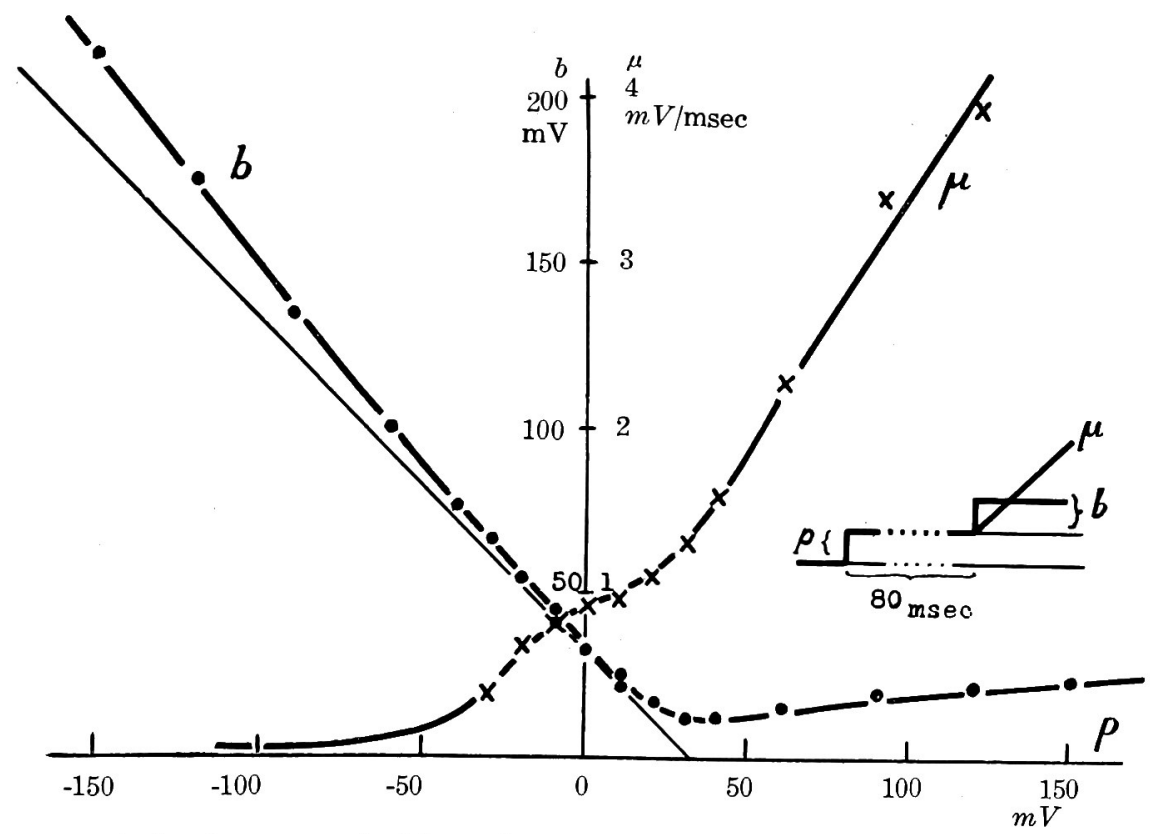

Fig. 6. Relation between polarizing voltage and rheobase or minimal gradient. Test pulses were started at about $80 \mathrm{msec}$. after make of the polarizing voltage. Toad's motor fiber at $6^{\circ} \mathrm{C}$.

We may define the magnitude of $E_{s}$, just as in the case of $E_{l}$, in such a way that in threshold excitation it barely attains unity. It is then obvious that a triangular voltage pulse of the final voltage $S$ produces $E_{s}$ having a maximum value of $S / S_{0}$, where $S_{0}$ signifies the threshold strength for this test pulse alone. If therefore a weak constant voltage pulse superposed on the triangular test pulse reduces the threshold for the test pulse to $S$, we find that the constant voltage pulse has produced, at the moment when the e.s. set up by the test pulse attains the maximum, the e.s. of the magnitude given by $\left(1-S / S_{0}\right)$.
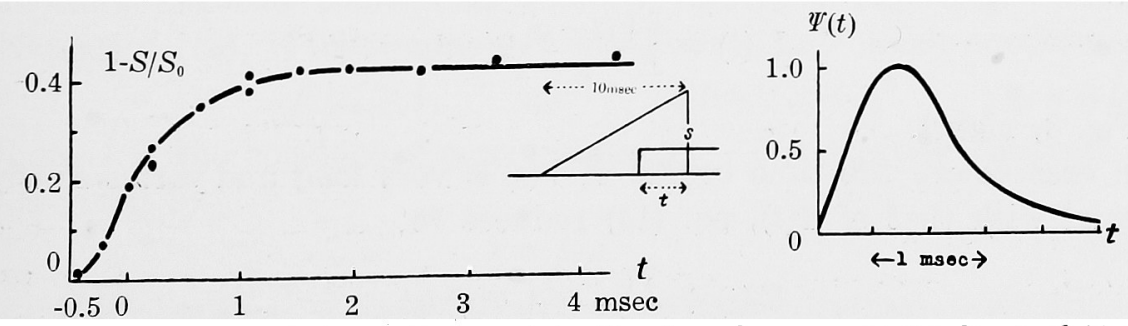

Fig. 7. Time course of the excitatory state $E_{s}$ set up by a constant voltage of $14.5 \mathrm{mV}$. Ordinate, the threshold reduction determined by a triangular test pulse of a duration of 10 msec. Abscissa, time from the onset of the constant voltage to the end of the test pulse.

Toad's motor fiber at $16^{\circ} \mathrm{C}$. (Tasaki, 1944, p. 205). 
Fig. 7 shows an example of the experiments in which the time course of $E_{s}$ set up by a weak constant voltage was traced by means of a triangular test shock. The fact that the observed threshold reduction $\left(1-S / S_{0}\right)$ was proportional to the strength of the conditioning, constant voltage indicates that the test of e.s. was done satisfactorily.

2.2 $E_{s}$ set up by a brief voltage pulse. It would be quite natural to assume the time course of e.s. shown in Fig. 7 to be constructed by integration of the elements $d E_{s}$ set up by brief pulses of which the continuous voltage pulse can be regarded as being comprised. Let us denote the time course of $E_{s}$ resulting from the action of a brief voltage pulse of strength $v$ and duration $d y$ by

$$
d E_{s}=\frac{v d y}{\delta} \Psi(t),
$$

where $\delta$ is a proportionality constant of the dimension of $m \mathrm{~V} \cdot \mathrm{msec}$. and $\Psi(t)$ a function of time, both characterizing the state of the tissue under investigation (compare with eq. 9 in Part I).

Then, the time course of $E_{s}(t)$ shown in Fig. 7 should be given by

$$
E_{s}(t)=\frac{v}{\delta} \int_{0}^{t} \varphi(y) d y
$$

(see Fig. 8, left).

Thus, comparing the first derivative of the curve in Fig. 7 with eq. (12) and considering that $\operatorname{Max} \Psi(t)=1$, we can determine the absolute value of the constant $\delta$ and the form of the function $\psi(t)$. The curve shown in Fig. 7, right, gives the time course of this function thus determined. The value of $\delta$ determined by this and other procedures was generally between 30 and $40 \mathrm{mV} \cdot$ msec.

2.3 The two threshold conditions in electric excitation. We are now prepared to describe all the experimental data stated in the preceding chapter in terms of the e.s. $E_{s}$. When the time course of a stimulating voltage is given by $V(t), E_{s}(t)$ set up by this voltage is expressed by the formula

$$
E_{s}(t)=\int_{0}^{t} \frac{V(t-y)}{\delta} \Psi(y) d y
$$

(see Fig. 8, right).

In case where the time course of $V(t)$ is very long and varies slowly compared with that of $\Psi(t)$, eq. (14) reduces to

$$
E_{s}(t) \doteqdot \frac{V(t-\zeta)}{\delta} \int_{0}^{\infty} \Psi(y) d y,
$$

where $\zeta$ represents the time required for $\varphi(t)$ to attain the maximum. In excitation of the fiber by a constant voltage of the rheobasic 
strength $\beta$, the plateau level of $E_{s}(t)$ should be equal to unity; hence, eq. (14) becomes
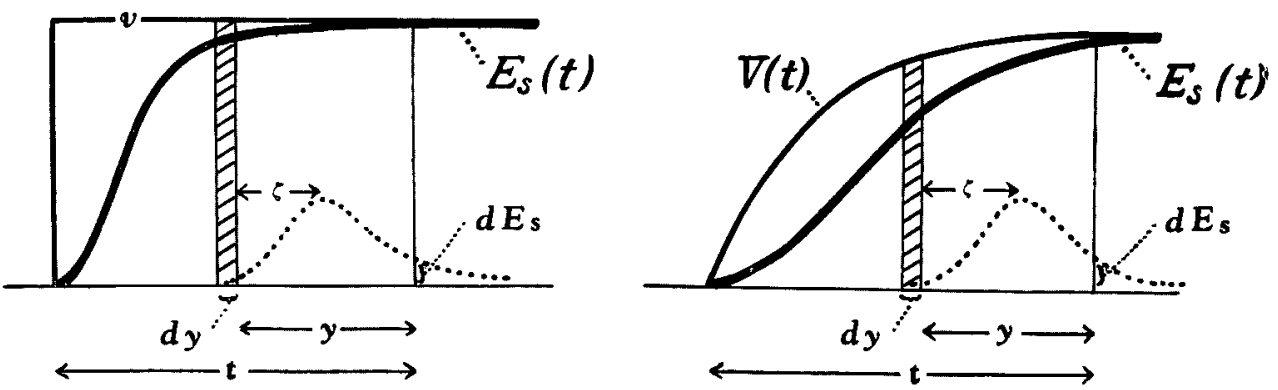

Fig. 8. Diagram illustrating the development of the excitatory state set up by a constant voltage and an exponentially rising voltage pulse. See text.

$$
1=\frac{\beta}{\delta} \int_{0}^{\infty} \Psi(y) d y
$$

Introduncing this relation into eq. (15), we obtain

$$
E_{s}(t) \doteqdot \frac{1}{\beta} V(t-\zeta)
$$

which roughly describes the development of $E_{s}$ set up by a slowly varying voltage $V(t)$. As $\zeta$ is considered to be of the order of $1-2$ msec. (see Fig. 7, right), we may further neglect this figure in eq. (17) if the stimulating voltage is long and slow enough; namely,

$$
E_{s}(t) \div \frac{1}{\beta} V(t)
$$

Comparing eq. (17) or (18) with the experimental result of Fig. 2, one easily finds that, only when the development of $E_{s}(t)$ takes place fairly rapidly, an impulse is released in accordance with the condition $E_{s}(t) \geqq 1$. It has been stressed in the preceding chapter that excitation occurs whenever the two conditions

$$
V(t) \geqq \beta, \quad \text { and } \quad d V(t) / d t \geqq \mu
$$

are simultaneously fulfilled by a slowly varying voltage $V(t)$. From the relation of (18), it follows that we should substitute these conditions with

$$
E_{s}(t) \geqq 1, \quad \text { and } \quad d E_{s}(t) / d t \geqq 1 / \lambda \text {, }
$$

where $\lambda$ is the constant of accommodation. ${ }^{1}$ The relation between $\lambda$ and $\mu$ is given by

$$
\lambda=\beta / \mu
$$

2.4 Condition to excite a node under catelectrotonus. We have conclused in the preceding section that the stimulating voltage $V(t)$ produces

1 It is also inferred from eq. $(19 \mathrm{~b})$ that $E_{b}(t)$ should accompany a constant of accommodation of its own which is smaller than $\lambda$. 
$E_{s}(t)$ in accordance with eq. (14), and that, if, at any moment, the two conditions (20) are simultaneously satisfied by $E_{s}(t)$, an impulse is released. In excitation by a constant voltage, $E_{s}(t)$ developes fairly rapidly and, as a consequence, the second condition (20b) is automatically satisfied whenever $E_{s}(t)$ approaches unity. In the case of exponentially rising voltages (Fig. 3), we had to take both of these two conditions into consideration.

We have seen above that, in a node of Ranvier under C.E.T., the value of $\mu$ is enormously great. It is then expected that fulfilment of the second condition (20b) becomes overwhelmingly difficult and, whenever we try to fulfil this condition, the first condition (20a) is automatically satisfied by $E_{s}(t)$. Under such circumstances, the threshold condition must have an extremely simple form.

From eq. (14), we obtain, by differentiation,

$$
\frac{d E_{s}(t)}{d t}=\int_{0}^{t} \frac{\partial V(t-y) / \partial t}{\delta} \Psi(y) d y .
$$

Hence, the threshold condition for the node under C.E.T., $d E_{s}(t) / d t=1 / \lambda$, should be of the form

$$
\int_{0}^{\ell} \frac{\partial V(t-y) / \partial t}{\delta / \lambda} \Psi(y) d y=1 .
$$

This equation expresses that, in a node under C.E.T., the voltage gradient $d V(t) / d t$ takes the part of $V(t)$ in the ordinary node in which the threshold condition is given by $E_{s}=1$. The statement that the excitatory effect of an electric stimulus is a function of only $d V(t) / d t$ naturally reminds us of the century-old law of du Bois Reymond (1848, cited by Lapicquie, 1926).

With a voltage increasing linearly at rate $g$, eq. (22) becomes

$$
\frac{d E_{s}(t)}{d t}=\frac{g}{\delta} \int_{0}^{t} \varphi(y) d y .
$$

When a constant voltage of the strength $v$ is made to start at $t=0$, eq. (22) becomes

$$
\frac{d E_{s}(t)}{d t}=\frac{v}{\delta} \Psi(t)
$$

The last two equations, together with the condition $d E_{s}^{\prime}(t) / d t=1 / \lambda$, indicate that, in excitation of a node under C.E.T., a linearly increasing voltage behaves like a constant voltage and a constant voltage like a brief shock for a normal node.

When two constant voltage pulses of the strength $u$ and $v$ are made to start at an interval $z$, the threshold condition becomes

$$
\operatorname{Max}\left\{\frac{v}{\delta / \lambda} \varphi(t)+\frac{u}{\delta / \lambda} \Psi(t-z)\right\}=1 .
$$


When a constant voltage of $u$ is applied at time $z$ after the start of a linearly increasing voltage of gradient $g$, the threshold condition is expressed by

$$
\operatorname{Max}\left\{\frac{g}{\delta / \lambda} \int_{0}^{i} \Psi(y) d y+\frac{u}{\delta / \lambda} \Psi(t-z)\right\}=1 .
$$

2.5 Experiments on the node under catelectrotonus. In the experiments of which an example is furnished in Fig. 9, a node of Ranvier on the distal side of a bridge-insulator was cathodally polarized by a constant voltage of $80 \mathrm{mV}$. Nodes on the proximal side of the insulator was narcotized with a 4 p.c. urethane-Ringer solution. After the polarizing voltage was allowed to act upon the fiber for 70 to $90 \mathrm{msec}$., constant voltages of varying strengths were superimposed on the polarizing voltage and the threshold voltage for this node was measured which was found to be about $15 \mathrm{mV}$ in this example.

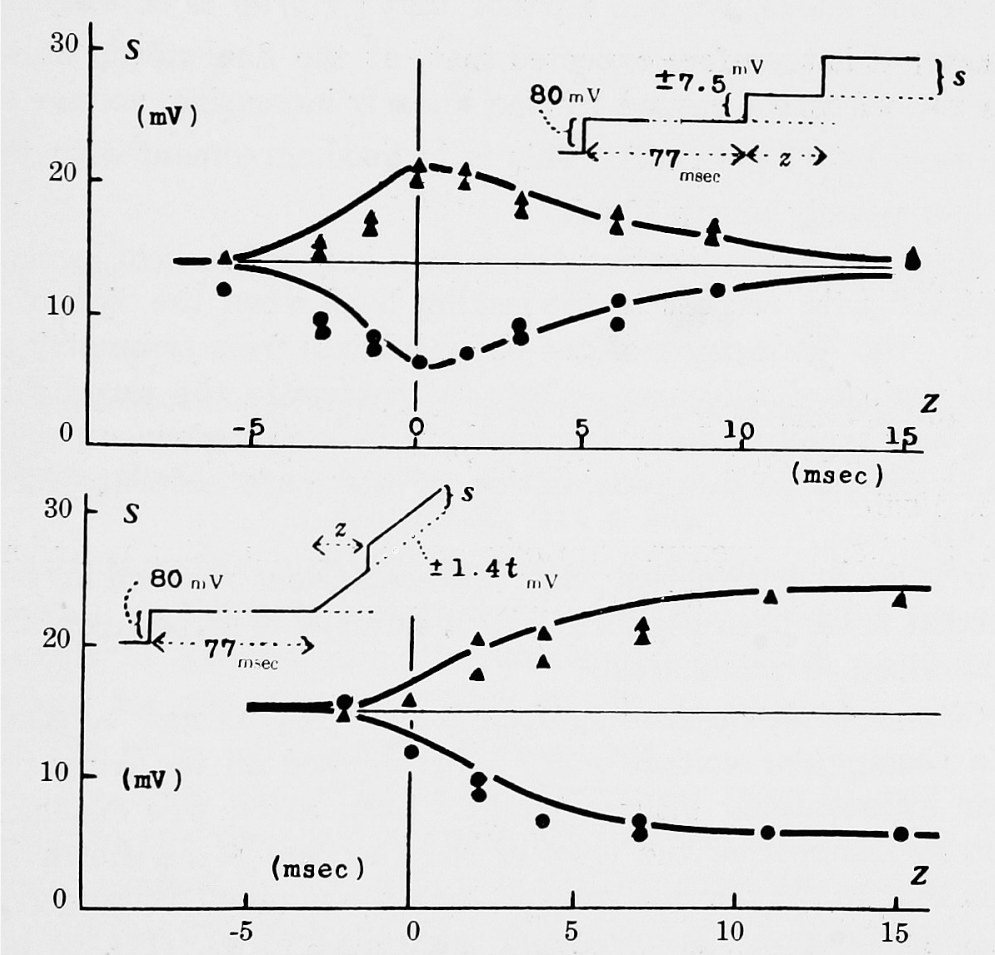

Fig. 9. Time course of the threshold variation caused by a rectangular voltage pulse (top) or a linearly increasing voltage (bottom) applied to a cathodally polarized node of Ranvier.

A rectangular voltage pulse was used for testing. Toad's motor nerve fiber at $6^{\circ} \mathrm{C}$.

'Then, a constant voltage of $\pm 7.5 \mathrm{mV}$ or a voltage increasing linearly at the rate of $\pm 1.4 \mathrm{mV} /$ msec. was started at the moment $77 \mathrm{msec}$. after the onset of polarization, and the change in the threshold level was traced by means of a testing constant voltage. In the figures, the threshold 
voltage $S$ for the test pulse is plotted against the time $z$ from the onset of the conditioning pulse.

Ii all the case, it was observed that the change in the threshold for the test pulse increases, at any moment, directly as the strength of the conditioning (either constant or linearly increasing) voltage. According to eqs. (26) and (27), the curves in Fig. 9, top, shoud be given approximately by ${ }^{1}$

$$
S=\delta / \lambda-v \Psi(t)
$$

and the curves at the bottom by

$$
S=\delta / \lambda-g \int_{0}^{l} \Psi(y) d y
$$

In fact, integration of the upper curve, corresponding to $v \Psi(t)$, gives a curve having the configuration of the lower one representing $v \int_{0}^{t} \Psi(y) d y$. From the upper curve, we find further that $\int_{0}^{\infty} \Psi(y) d y$ is of the order of 7.5-8 msec. $^{2}$ It is therefore expected that, at the final steady state, the change in the threshold caused by the linearly increasing voltage should be $g \int_{0}^{\infty} T(y) d y=1.4 \times 8 \doteqdot 11 \mathrm{mV}$. This is in good agreement with the observed value, namely about $10 \mathrm{mV}$.

Eqs. (28) and (29) predict that the change in the threshold level should be symmetrical with respect to the resting level when the sign of $v$ or $g$ is reversed. The divergence of the observed data from symmetry occurs only in the regions of the curves where the change in the threshold level progresses very rapidly. It is obvious that, in such regions, eq. (28) and (29) can not give a good approximation of the more accurate formulae (26) and (27).

It should be added further that the conclusions reached in the preceding section have been supported by a number of experiments which are not described in this article.

2.6 Change in the rheobase by electrotonus. Let us now consider the effect of a rectangular voltage pulse superimposed on the early plateau level of an exponentially rising voltage. Soon after this exponentially rising voltage has crossed the level of the rheobase $\beta$, as shown by the diagram in Fig. 10, at the rate smaller than the minimal voltage gradient $\mu$, the magnitude of e.s. is considered, according to eq. (17), to be well above unity. ${ }^{3}$

1 Cf. the derivation of eq. (6) of Part I, and note that $u=S$ and $\operatorname{Max} \Psi(t-z)=\Psi(\zeta)=1$. In 128 ) and (29), $z+\zeta$ is replaced for simplicity with $t$.

2 Note again that the maximum value of $\Psi(t)$ is equal to unity.

3 Application at this stage of another exponentially rising voltage of varying time constants actually give a v-re straight line passing near the origin, but having a slope steeper than that in the normal nerve. 
If a weak constant voltage pulses is added at this stage, the e.s. rises rapidly and the second condition $d E_{s}(t) / d t \geqq 1 / \lambda$ may be fulfilled very readily. Since the first condition $E_{s}(t) \geqq 1$ has already been satisfied by the e.s., it is expected that a very weak rectangular pulse is sufficient to release an impulse at this stage.

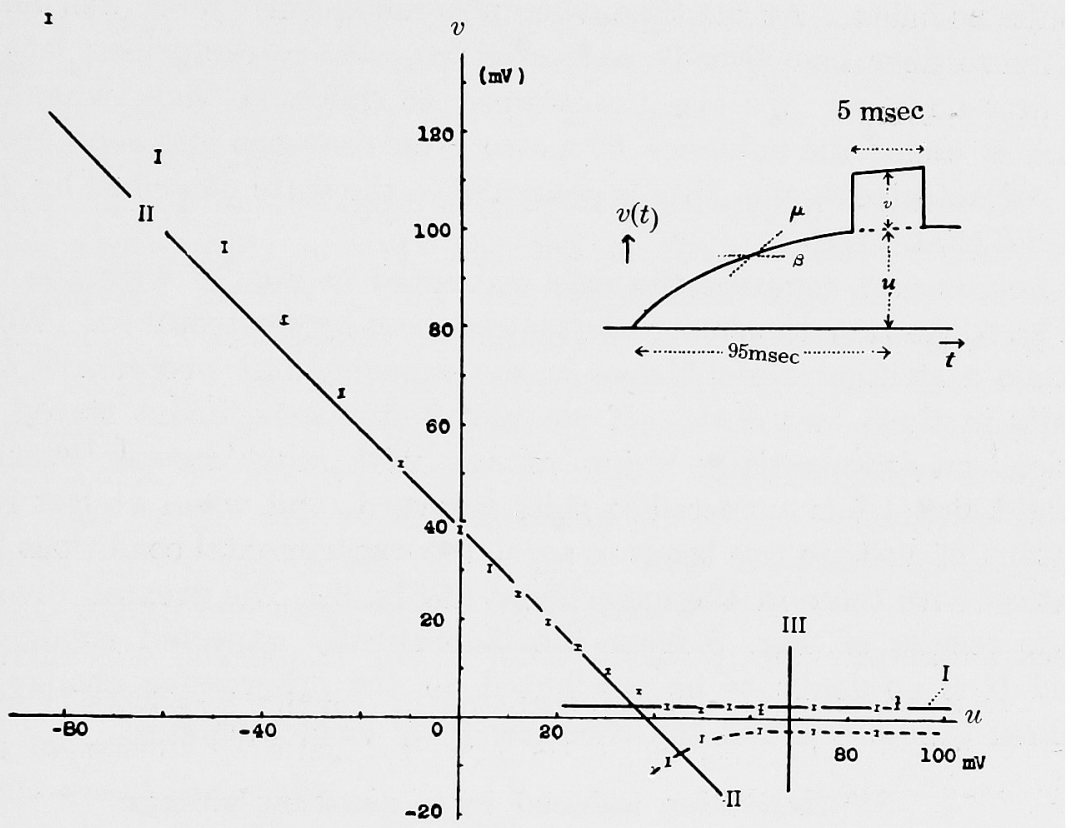

Fig. 10. Relation between strength of an exponentially rising voltage $r c=0.1 / \mathrm{sec}$ and threshold strength of a rectangular pulse superimposed at $95 \mathrm{msec}$. after the onset of the slowly rising voltage. Toad's motor nerve fiber at $16^{\circ} \mathrm{C}$. Straight line II intersects both axes at $45^{\circ}$. (Tasaki, 1944, p. 219).

If a rectangular pulse of the reversed direction is superimposed at this stage, it is similarly expected that the two conditions for excitation should easily be satisfied soon after the end of the pulse. And the threshold voltage for the reversed pulse should be approximately equal to that for the pulse with normal positive sign.

Fig. 10 shows the result of an observation done to test this point. In this figure the threshold strength $v$ of the rectangular pulse is plotted as ordinate against the strength $u$ of the exponentially rising voltage as abscissa. In this preparation, the rheobase was about $38 \mathrm{mV}(v$ for $u=0$ ). When $u$ was above this resting rheobase, the threshold for the rectangular pulse was found to be $1.5-3 \mathrm{mV}$. The straight line I in the figure therefore represents excitation by the mechanism stated above. The broken line in the figure is also what is expected in the argument above.

The straight line II represents the relation $u+v=48$. It is obvious that, when $u$ is below the resting rheobase $\beta$, the threshold is determined by the first condition $E_{s}=1$, or $u+v=\beta$, including the negative side of $u$. 
A point of interest in this experiment is that the straight line I is extending to the region where the exponentially increasing voltage is in itself effective to release an impulse. The straight line III in the figure represents the boundary between sub- and super-threshold $u$ values. In such cases, the threshold value of $v$ was determined taking the second impulse as index. At the time when this rectangular pulse was delivered, the nerve fiber had already recovered from the refractoriness left behind the first impulse. We are thus forced to conclude that, when a fiber which is under the influence of a strong current has just recovered from the refractoriness, the fiber is generally in the state described by $E_{s}(t) \geqq 1$ and $d E_{s}(t) / d t<1 / \lambda$.

Let us now compare the data presented in Fig. 10 with the curve $b$ in Fig. 6 showing the change in the rheobase by electrotonus. We notice at once a striking resemblance in the experimental procedures and the results in these two cases. If we use, in the experiment stated in this section, an exponentially rising voltage with much steeper ascent, the straight line III is expected to shift leftward, and when at last its time constant of voltage rise tends to zero, the experimental conditions become identical with those in the experiment of Fig. 6 . The greater divergence of the results of Fig. 6 from the theoretically expected straight lines I and II is no doubt to be attributed to the progressive change in the minimal gradient $\mu$ and the rheobase $\beta$ by electrotonus.

\section{Repetition induced by a constant voltage}

3.1 The mechanism of repetition. When a nerve fiber is subjected to a constant voltage of suprarheobasic strength, it is sometimes observed that the fiber responds with more than one impulse. This occurs, as is well known, only when the minimal gradient of the fiber is fairly small. We now proceed to describe the mechanism of such a repetition in terms of the excitatory state $E_{s}(t)$.

As has been stated already, the development of $E_{s}^{\prime}(t)$ under the influence of a constant voltage $v$ is described by the formula

$$
E_{s}(t)=\frac{v}{\delta} \int_{0}^{\ell} \Psi(y) d y
$$

As soon as this e.s. satisfies the first condition for excitation, $E_{s}(t) \geqq 1$, a nerve impulse is released, since the second condition $d E_{s}(t) / d t \geqq 1 / \lambda$. is in this case automatically fulfilled (see 2.4). Then the nerve fiber falls in refractory phase.

During the absolutely refractory phase, the value of $\delta$, the minimal quantity required for production $E_{s}$ of the unit magnitude, is of course infinite; therefore, according to eq. (13), the e.s. is considered to become zero. As the excitability recovers during the relatively refractory phase, $\delta$ recovers gradually and accordingly the e.s. is considered to increase 
again. In the consideration of e.s. during the refractory phase, we must regard $\delta$ as a function of the time $t$. Hence, replacing $\delta$ in eq. (13) with $J(t)$,

$$
E_{s}(t) \doteqdot \frac{v}{J(t)} \int_{0}^{\infty} \varphi(y) d y
$$

or, more simply,

$$
E_{s}(t) \doteqdot v / \beta(t) \text {. }
$$

We have already seen that $\delta / \int \Psi(y) d y$ is nothing but the rheobase $\beta$ for the node (eq. 16). During the refractory phase, the rheobase is known to vary like the ordinary excitability determined by a brief shock (cf. Tasaki and others, 1948).

When the constant voltage $v$ is super-rheobasic, it is clear that the e.s. rises above unity towards the end of the refractory phase. But, as the recovery process is slow and continuous, the second condition $d E_{s}(t) / d t \geqq 1 / \lambda$ can not always be fulfilled by $E_{s}(t)$ in that occasion (cf. the preceding section). The smaller the minimal gradient and the stronger the constant voltage, the more readily will be the second condition satisfied. Thus, we are led to the conclusion that the second impulse is released only when the second condition for nerve excitation is also fulfilled by $E_{s}(t)$ given by eq. (30) or (31). And the same can be said of the third, fourth and other following impulses.

During the passage of the constant current, the minimal gradient and the rheobase is known to increase gradually. Thus, when it has become impossible for e.s. to satisfy one of these two conditions, the nerve fiber ceases to fire impulses.

3.2 The effect of an
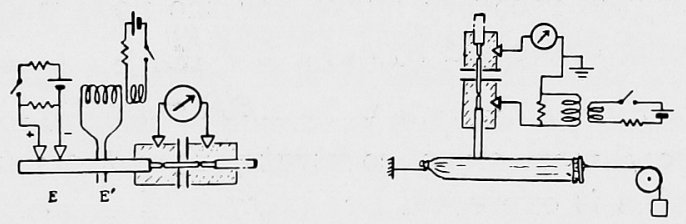
extra-impulse upon the rhythm of discharge.

According to the interpretation of the mechanism of repetition stated above, the e.s. at the node under the influence of a super-rheobasic constant voltage reduces to

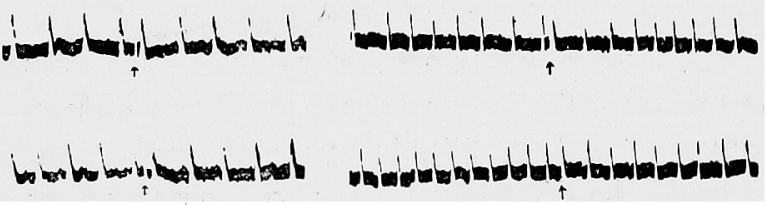

Fig. 11. Left: Effect of an "extra-impulse" upon the rhythm of repetition induced in a toad's motor nerve fiber by a constant voltage of 6 volts. Time marks, $10 \mathrm{msec}$. zero whenever the node apart. Right: Similar effect upon the impulse discharge fall in the refractory from a tonic stretch receptor in the sartorius muscle of phase. If therefore such a the toad. $11^{\circ} \mathrm{C}$.

node repetitively discharging impulses is excited by an "extra-stimulus", the e.s. at this node should be extinguished at once, and as the excitability recovers gradually along its recovery curve, it should augment again. Hence, the next impulse ought to be released at a regular interval after the "extra-impulse". 
In the experiment of Fig. 11, left, a sciatic nerve taken from a spring toad was subjected to a super-rheobasic constant voltage and the repetitive response in a single motor nerve fiber was recorded with an oscillograph of the Duddel type. During the course of repetition, a single brief shock was applied to the nerve and the effect of an "extraimpulse" thus induced upon the rhythm of repetition was investigated. As can clearly be seen in the records, the time-interval from the extraimpulse to the next was approximately equal to the regular interval, regardless of the interval between the extra-impulse and the preceding one.

Such an effect of the extra-impulse upon the rhythm of repetition has been demonstrated on the pace-makers induced by " natural stimuli". Gilson's experiment (1928) on the frog heart, the experiment of Fig. 11, right, on the tonic muscle afferent, which was done to re-examine Matthews' result (1933) and some other observations suggests very strongly that the mechanism of repetition is probably the same in all the tissues.

3.3 The strength of the constant voltage and the frequency of repetition. According to eq. (31), the time $T$ required for e.s. to recover to unity after the node has once been thrown into action is given by

$$
\beta(T)=v .
$$

If the recovery curve for the node under investigation is assumed as being expressed by

$$
\beta(t)=\beta_{0} e^{1 / \kappa},
$$

where $\beta_{0}$ and $\kappa$ are constants, then the interval $T$ between successive impulses is expressed by

$$
1 / T=\kappa \log \frac{v}{\beta_{0}} .
$$

Although this formula relating the voltage $v$ and the frequency of repetition $1 / T$ is supported to some extent by direct observation (see Fessard, 1936, p. 35), it must be born in mind that the constants $\beta_{0}$ and $\kappa$ should be different from those obtained by direct determination of the recovery curve on the resting node, since the recovery process is retarded by C.E.T.

3.4 Progressive change of the state of the node by electrotonus. The most remarkable change brought about by C.E.T. in the nerve fiber is the increase in the minimal voltage gradient. The rate of increase varies directly as the polarizing voltage. Under the influence of a constant voltage, the minimal grandient increases first rapidly and then gradually within 0.2-0.5 sec. from the onset of the voltage; but, even after a minute, there is a gradual increase in it. A.E.T. readily reduces the minimal gradient to a value too small to be determined accurately. 
In dealing with the rheobase of a node under electrotonus, it is necessary to mention explicitly whether or not the polarizing voltage is regarded as a part of the stimulating voltage. In the analytical treatment of the rheobase of the node under A.E.T., the sum of the polarizing voltage and the rectangular voltage was regarded as the effective stimulus (Fig. 10). In the case of C.E.T., however, the observed threshold for the superimposed rectangular voltage pulse is determined partly by the second condition $d E_{s}(t) / d t \geqq 1 / \lambda$ and consequently the change in the value of $\beta$ can not be determined directly in that case. The change in the observed rheobase by a very strong C.E.T. is undoubtedly attributable, at least partly, to the rise of the minimal gradient in that case. During A.E.T., $\beta$, the sum of the polarizing voltage and threshold strength for the test pulse, shows a slight and gradual increase (see the divergence of the curve $b$ from the straight line in Fig. 6).

Turning now to the effects of these changes upon the process of repetition induced by a constant voltage, the gradual increase in the minimal gradient by C.E.T. is considered to be the cause of the cessation of repetition. It seems possible to interpret Katz's result on the duration of repetition (1936) in terms of the change in the minimal gradient by C.E.T.

The rise of $\beta$ by A.E.T. stated above predicts a curious phenomenon : If one apply, to an anodally polarized node, a constant voltage of a barely subthreshold strength, which is greater than the sum of the polarizing voltage and the resting rheobase, it is expected the node under investigation is brought gradually from the state of A.E.T., i.e. the state of increased $\beta$, into normal and then to the state of C.E.T. And, when the node passes the state of normal $\beta$, the stimulus must become superrheobasic and make the node fire impulses. Actual observation this phenomenon indicated that, in a delayed repetition of this type, impulses can be set up more than 0.1 sec. after the make of the constant voltage. A detailed description of this phenomenon is reserved for a subsequent paper.

In the operated region of a single nerve fiber preparation of the toad, it is extremely difficult to induce a nerve impulse by the break of a constant voltage pulse. It seems certain that the break excitation observed in the whole nerve trunk results from excitation by the countere.m.f. set up by the imposed current at the surrounding tissue in the nerve trunk.

\section{Conclusion}

From all what has been mentioned in this article, it may be concluded that the use of the term "excitatory state" serves for a systematic, coordinative description of various events which follow the application 
of an electric stimulus and precede the initiation of a nerve impulse. The most striking feature of the excitatory state, besides its property to release an impulse at a certain critical level, is its behavior to follow the "law of proportionality and superposition":

The excitatory state varies directly as the applied voltage; and, when two voltage pulses act upon a node concurrently, the excitatory state produced by this complex stimulus is given by the algebraic sum of the excitatory state resulting from the action of the individual pulses.

This appears to indicate that the differential equation describing the developement of this state is linear. It can be a partial differential equation, the strength of stimulus being introduced as a perturbation term or as the boundary condition.

One might argue, against the scheme of the description in this article, that we have been dealing only with the propagated impulse and not with the minimum response localized at a node of Ranvier. Although the presence of a local response is a logical consequence of electric transmission of the nerve impulse, there is good reason to believe that a direct demonstration of a graded response in a normal node is extremely difficult. Even when a node developes an action current of only one sixth the normal size, the activity at this node can throw the next node into action.

If the electric conductance accross the active, depolarized region of the plasma membrane is overwhelmingly great compared with that across normal, resting regions, it is expected that even a minute active patch of the membrane may eventually be able to bring the surrounding resting region into action. Under such circumstances, we may put aside the problem of local excitation of a node for a moment and may speaks of the excitatory changes at a point of the nerve fiber. It appears to me that the problem of local response complicates the matter only when the safety margin of the fiber is reduced significantly, possibly by an injury from the operation or by contact with a metal electrode.

\section{References}

Cardot, H. and Laugier, H. 1913 J. Physiol. Pathol. gén. 15 : 1134-1147.

du Bois Reymond, R. 1848 Untersuchungen über die tierische Electricität, Berlin.

Fessard, A. 1936 Propriétés rhythmiques de la matiere vivante I, Paris.

Gilson, A. S. 1936 Amer. J. Physiol. 116 : 358-366.

Granit, R. 1947 Sensory mechanism of the retina, Oxford.

Hill, A. V. 1936 Proc. Roy. Soc. B. 119 : 305-355; 440-453.

Lapicque, L. 1926 L'excitabilitè en fonction du temps, Paris.

Matthews, B.H.C. 1933 J. Physiol. 78: 1-53.

Schriever, H. 1932 Z. Biol. 93: 249-258.

Solandt, D. Y. 1936 Proc. Roy. Soc. B. 119 : $355-379$; 120 : 389-408.

Suzuki, M. 1937 Pflügers Arch. ges. Physiol. 239 : 81-96.

Tasaki, I. 1939 Amer. J. Physiol. 125 : 380-395.

- 1942 Pflügers Arch. ges. Physiol. 245 : 665-679.

- 1944 The physiology of the nerve fiber (in Japanese), Tokyo.

- 1949 Biochim. Biophys. Acta 3: 498-509.

-, Mizuguchi, K. and Tasaki, K. 1948 J. Neurophysiol. 11 : 305-310. 